\title{
A Study of Address Shortage in a Tree Based ZigBee Network for Mobile Health Applications
}

\author{
Ka Lun Lam ${ }^{1}$, Hoi Yan Tung ${ }^{1}$, King Tim Ko ${ }^{1}$, Kim Fung Tsang ${ }^{1}$, \\ Hoi Ching Tung, ${ }^{1,2}$ Yat Wah Leung ${ }^{3}$, Wing Hong Lau ${ }^{1}$ \\ ${ }^{1}$ Department of EE, City University of Hong Kong, Hong Kong, China \\ ${ }^{2}$ School of Engineering \& Mathematical Science at City University London, London, UK \\ ${ }^{3}$ Department of SEEM, City University of Hong Kong, Hong Kong, China \\ Email: \{kalunlam, hytung\}@student.cityu.edu.hk, \{eektko, ee330015\}@cityu.edu.hk, Cherry.Tung.1@city.ac.uk, \\ 50997159@student.cityu.edu.hk, itwhlau@cityu.edu.hk
}

Received February 7, 2012; revised March 19, 2012; accepted April 2, 2012

\begin{abstract}
There are increasing demands for mobile health applications. This paper reports the development of a mobile health profile which dedicates to mobile applications. The mobile health profile is developed in association with the ZigBee Health Care profile and the IEEE 11073 standard which is normally applied to non-mobile applications. Since mobile sensors have to be carried by patients, the mobile health profile must facilitate mobility. In this investigation, a ZigBee fixed-mobile network (ZFMN) is defined and developed to supplement the ZigBee Health Care Profile for patient monitoring. The mobility study of ZigBee is performed using a random waypoint OPNET simulation model. In a ZFMN, the critical issue of address shortage is identified and discussed. It is analyzed that the problematic address shortage in a ZFMN may generate a huge amount of orphaned end devices and thus the packet drop percentage may potentially rise to $70 \%$, rendering the network unable to function properly. Without introducing additional governing schemes, it is evaluated that the communication of the entire ZigBee network may paralyze. Further vigorous test are performed (by OPNET) on the communication capability of ZFMN when devices are randomly moving and sending data in $1 \mathrm{~s}$. It is vital to point out that under the adverse condition of address shortage, the performance of a ZFMN is still encouraging as long as the packet drop percentage has been kept below $3 \%$ before running out of address. The conclusion drawn in this analysis is that the packet drop percentage should be kept below 3\% to provide a satisfactory QoS for an effective mobile health application using ZFMN such as patient monitoring. Such finding is also important for other future mobile application design of ZigBee. The address shortage issue is left as an open problem that needs attention for a resolution.
\end{abstract}

Keywords: Mobile Health; Patient Monitoring; Mobility; ZigBee; Tree Address Assignment; Address Shortage

\section{Introduction}

The ZigBee protocol was originally defined for non-mobile applications and has gained increasingly importance recently. Pilot works on ZigBee did not discuss the design of large scale system which demands mobility. There are increasingly more applications which demand mobility within a large ZigBee fixed network, say for patient monitoring application in hospitals and agedperson caring centers. Up to present, there has been no study on enabling mobility in a fixed ZigBee network. It will soon be explained that such mobility enabling is different from the traditional ZigBee ad hoc networks in terms of topology and addressing requirement.

Aging tends to be a serious worldwide problem in

${ }^{*}$ The work is supported by CityU I2R Funding Scheme (6351010). coming 20 years. Health care for aged persons will be a very alarming issue. It is insightful to understand the pragmatic application of ZigBee by studying the demands of future health care for inpatients and outpatients within hospitals. Let us envisage the required environment in hospitals in the future as follows. In the hospital, mobile sensors e.g. blood pressure monitor, pulse oximeter and blood glucose meter will be carried by patients. The health status from these sensors will be regularly collected and sent to the centralized patient monitoring server. Under such a circumstance, the server and routers typically needs to communicate with a pool of mobile devices to ensure a robust communication between mobile sensors and the centralized server. The application scenario just described is referred as mobile health. There is no doubt that mobile health for patient monitoring will 
be deployed in the future to help relieving the burden of hospitals and thus rendering the application important.

In essence, in mobile health applications, the selfhealing capability in a non-mobile network is a perfect feature to be sought for. In this investigation, based on the salient mesh capability of ZigBee, a new ZigBee network structure is proposed that caters for the selfhealing requirement and also provides efficient mobility. An efficient mobility will satisfy the needs for future in-patient monitoring systems.

It was explained earlier that ZigBee was originally not designed for mobility. Moreover, the ZigBee ad hoc network recently developed does not provide a fully dedicated and reliable communication path due to its ever-changing topology [1-3]. By virtue of the mesh capability of ZigBee, the ZigBee protocols appear to be the right candidate. In this work, the provision of mobility in a non-ad-hoc ZigBee network is achieved by modifying and converting a part of the fixed network to facilitate mobile and yet non-ad-hoc characters. The potential huge demands for health care (under healthcare initiative) drive the need for a standard protocol, namely the ZigBee Health Care Profile [4-6], for health applications. With such positive input from the industries, researchers are keen on applying ZigBee technology to various medical applications. In the profile, ZigBee integrates with medical sensors to enable communication between patients [4-6]. Despite there were feasibility studies [7-11], no study focused on the addressing assignment scheme for moving nodes in a non-ad-hoc manner. Thus existing ZigBee protocol structure will not be able to cope with the need and resolve any former conflicts. Such a mobile health network is referred as a ZigBee Fixed-Mobile Network (referred as ZFMN in the following context) in differentiation to the former ZigBee ad hoc network and fixed ZigBee network. It is reiterated that the ZFMN is inherently not an ad hoc network in which the network topology is typically continuously changing. It will be shown in the following context that the mobility need is not addressed in the ZigBee Health Care Profile. In mobile health applications, some end devices are nomadic and always move from time to time due to the intrinsic need in the application, these nodes are referred as mobile nodes or mobile end devices. These mobile nodes have to make connection with multiple parents with multiple addresses due to the continuous handover. It will be shown in this paper that there will be an address shortage due to the ever-changing parent character in a ZFMN. The performance inadequacy in a ZFMN will be quantified and a design rule will be given.

A general network structure is shown in Figure 1. The network consists of a coordinator, multiple routers and end devices. There is only one coordinator in each net- work. The coordinator is responsible for the network formation. During the network formation, the network channel, Personal Area Network Identifier (PAN ID) and extended PAN ID is chosen by the ZigBee coordinator. The coordinator acts as a trust center in the network. The routers and end devices can be medical devices such as oxygen saturation monitor, blood pressure monitor, Electrocardiography (ECG), and non-medical devices such as gateway, light and surveillance system etc. In ZFMN, the coordinator and routers are deployed at a fixed location while end devices serve as mobile nodes. Consider the example illustrated in Figure 1, when the oximeter (end device) moves out of the coverage of router $\mathrm{C}$ and reaches the coverage of router A (referred as handover), the oximeter then requests the address from router A (indicated as "Cmd 1" in Figure 1). Then, router A assigns an address to the oximeter (indicated as "Cmd 2" in Figure 1). From this point in time onward, router A will then become the parent of the oximeter. In a ZigBee ad hoc network, the ever changing topology resets from time to time the address assignment during the network formation. On the other hand, in a ZFMN, the amount of address is pre-assigned to each parent node due to the inherent nature of a constant network topology, thus no reset will be performed in a ZFMN network. Moreover, in contrast to a ZigBee ad hoc network, the addresses of end devices in a ZFMN will be continuously changing and thus presenting a challenge to the network yet to be resolved. Thus, the mobility character (and thus treatment) in a ZFMN will be very different to the mobility character in a ZigBee ad hoc network. This paper is to conduct a study of the characteristics of the ZFMN.

\section{ZigBee Mobility Solution}

The ZigBee Health Care Profile only focuses the protocol integration of IEEE 11073 (Standard communication

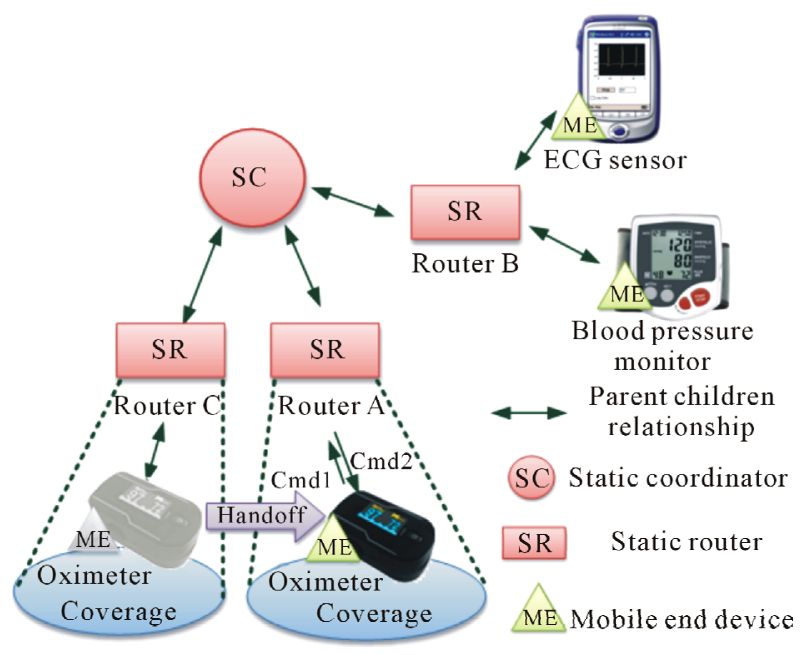

Figure 1. ZigBee Fixed-Mobile Network (ZFMN). 
protocol of health care device) and ZigBee but offers no capability to the mobility management of ZFMN. In mobile health, mobile devices are needed so that patients carrying sensors may be reshuffled to other wards with convenience and accuracy. Thus a ZFMN that provides mobility and supports patient monitoring service is needed. The ZFMN so defined is now designed as an application that interoperates with IEEE 11073 and the ZigBee Health Care Profile. The relationship of ZFMN, IEEE 11073 and ZigBee Health Care Profile is illustrated in Figure 2.

There is no doubt that such a structure is definitely a catalyst to the development of ZigBee Personal Home \& Hospital Care applications. For example, the panic button for senior citizen is one of the typical examples of potential ZigBee applications in mobile health. When the senior citizen is not feeling well, he/she may press the on-body panic button, and the call center will receive the help signals and so timely aids/services can be offered. More importantly, ZigBee Personal Home \& Hospital Care applications demand that the body status of users must be monitored from time to time. In essence, when monitoring devices detect abnormal readings from a user, warning messages will be sent to the hospital through the ZFMN. Therefore, it is critical for ZigBee to support the mobility of end devices which in turn facilitates freedom of movement for patients. For example, a patient may carry ZigBee personal health devices (indicating as end device in Figure 1), such as blood pressure monitor, glucose monitor and thermometer, walking around in the retirement center (hence mobile nodes). During the process, the end devices may loss connection with their parent devices (indicating as router in Figure 1) due to the patient movement. To enable seamless handover and reliable connection, end device will handover to another router. This implies that a router or an end device moves with low-speed (e.g. walking) in a ZFMN - this is a new development of healthcare application. However, the network performance of a new ZigBee mobile network is deficient. To provide insight for the mobility design of ZigBee, this paper investigates the performance of the

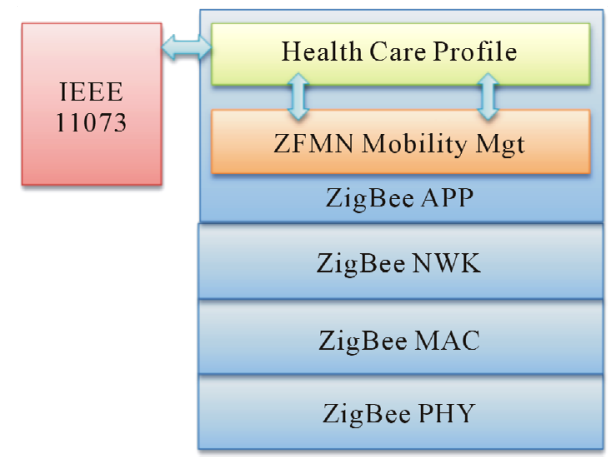

Figure 2. ZFMN mobility management. new ZigBee mobile network and identifies the current design deficiency.

\section{Address Assignment}

When an end device joins the network, a network address is requested by the parent. The parent can be a router or a coordinator. Traditionally, Tree Address Assignment (TAA) scheme and Stochastic Address Assignment (SAA) scheme are supported by the ZigBee network. SAA assigns the device address randomly to enable an efficient use of address. In SAA, the same address may be assigned to two devices. Hence SAA requires extra cost in address conflict resolution and routing [12,13]. Such problem is referred as the address conflict. In view of the limited capacity of ZigBee devices, SAA is typically expensive in terms of resource requirement. Based on the economical nature of SAA, focus is drawn to the development of the TAA scheme in this paper.

TAA aims to provide a finite number of network addresses to a potential parent. When a device joins the network, the parent assigns a unique address to the device. The parent will assign address according to the pre-defined parameter predetermined by the ZigBee coordinator as shown in (1).

$$
\mathrm{A}_{\mathrm{d}, \mathrm{n}}=\mathrm{A}_{\mathrm{p}}+\mathrm{n}+\left(\frac{1+\mathrm{Cm}-\mathrm{Rm}-\mathrm{Cm} \times \mathrm{Rm}^{\mathrm{Lm}-(\mathrm{d}-1)-1}}{1-\mathrm{Rm}}\right) \times \mathrm{Rm}
$$

where $\mathrm{d}$ is the network depth and $\mathrm{d} \in\{1,2, \cdots, \operatorname{Lm}\} ; \mathrm{n}$ is the $\mathrm{n}^{\text {th }}$ end device that joining the network with $\mathrm{n} \in\{1,2, \cdots, \mathrm{Cm}-\mathrm{Rm}\}$ and $\mathrm{A}_{\mathrm{p}}$ is the address of parent.

The developer determines the maximum number of children for each parent $(\mathrm{Cm})$, the maximum number of router for each parent $(\mathrm{Rm})$ and the maximum depth in the network $(\mathrm{Lm})$ according the ZigBee network structure and the coordinator stores the captioned parameters ( $\mathrm{Cm}, \mathrm{Rm}$ and $\mathrm{Lm}$ ) for network initialization. A design example will be discussed in Section 4.

\section{Investigated Scenarios}

To investigate the performance, a ZFMN simulation model is evaluated by using OPNET [14]. The simulation model consists of a coordinator, three routers, $\mathrm{k}$ mobile end devices, and a mobility model. In the simulation, three routers, one coordinator and $\mathrm{k}$ end devices form a tree structure in a ZFMN. Despite the fact that a ZFMN is not an ad hoc network (in terms of address assignment and topology), it must be pointed out that as far as mobility is concerned, end devices move in a manner similar to an ad hoc network which is satisfactorily described by the random waypoint mobility model that is widely used in mobility studies [15-18]. To evaluate the influence of 
the number of end device (mobile device), $\mathrm{k}$, on the ZFMN, the performance is evaluated under different scenarios and details will be given in the later context. The network structure of the simulation models is illustrated in Figure 3 and the simulation model parameters are shown in Table 1.

The TAA as defined by the ZigBee standard may favorably be applied to fixed networks but not ZFMNs. The contribution of this work is to investigate the performance of TAA under a ZFMN which may be widely adopted in the future. It was described that the number of available address is important in the address assignment in a ZFMN, such address availability is referred as the capacity of an end device, Ec. Hence Ec will be an important parameter to be discussed in a ZFMN. It is obvious that Ec depicts the number of free address of a parent. In the initialization of a ZFMN, Ec equals the difference between $\mathrm{Cm}$ and $\mathrm{Rm}$ and is given by:

$$
\mathrm{Ec}=\mathrm{Cm}-\mathrm{Rm}
$$

When an end device has joined the parent, Ec of the parent is reduced by 1 .

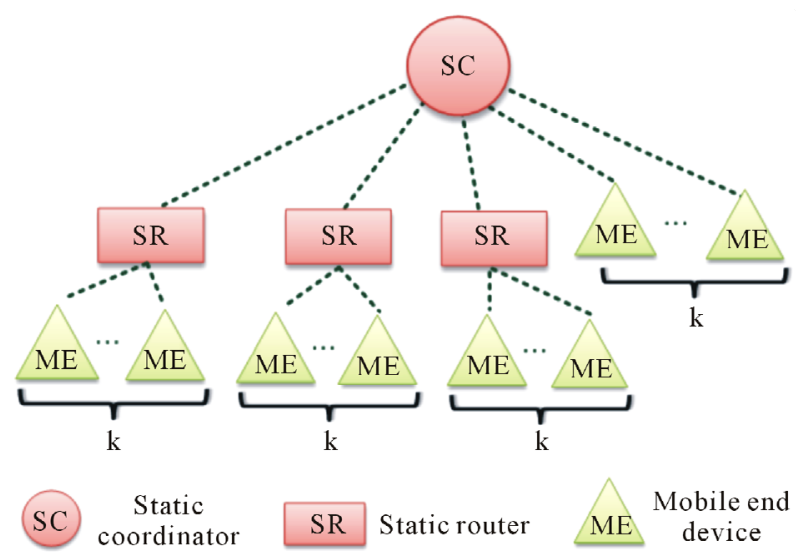

Figure 3. Network structure of simulation model.

Table 1. Simulation model.

\begin{tabular}{cc}
\hline Parameter & Setting \\
\hline Number of Addresses (Na) & 80 \\
Number of End Devices (k) & $4-80$ \\
Maximum Children (Cm) & 23 \\
Maximum Router (Rm) & 3 \\
Maximum Depth (Lm) & 4 \\
Address Assignment Scheme & Tree \\
Mobility Model & $1 \mathrm{~m} / \mathrm{s}$ \\
Moving Speed & $600 \mathrm{~s}$ \\
Simulation time (Ts) & $1 \mathrm{~s}$ \\
Packet Inter-arrival time (Ti) &
\end{tabular}

A square shaped area of 20,000 square feet is a very common size for commercial use and this is in line with the recommendation from ZigBee Alliance [3]. Also, it is typical that $\mathrm{Ec}<20$ [19] and hence the router in a ZFMN will not handle more than 20 mobile end devices. To cater for mobility in this 20000 square feet area, four (4) static devices, including one coordinator and three routers (i.e. $\mathrm{Rm}=3$ ), are deployed to cover the whole area (refer to Table 1). As a result, 80 (i.e. 20*4) end devices addresses are required in this ZFMN. The maximum number of children $(\mathrm{Cm})$ for each parent is given by the sum of maximum number of router $(\mathrm{Rm}=3)$ and $(\mathrm{Ec}=$ 20) (i.e. $\mathrm{Cm}=\mathrm{Rm}+\mathrm{Ec}=23$ ). To investigate the impact of $\mathrm{k}$ on the network performance, twenty (20) scenarios are investigated ( $\mathrm{k}$ varies from 4 to 80 , in step of 4 , i.e. $\mathrm{k}$ $=4)$. The reason for selecting $\mathrm{k}=4$ is because there are four (4) static devices in the ZFMN (1 coordinator +3 router). In the design, $\mathrm{Lm}$ is confined to 4 to ensure the end to end latency is within $1 \mathrm{~s}$. Without lose of generality, in the simulation, end devices (which are exponentially distributed) are best described to move in accordance with Random Waypoint mobility model with 1 meter/second average moving speed. Typically there are substantial handover in $600 \mathrm{~s}$, hence the simulation time for each scenarios is configured as $\mathrm{Ts}=10$ minutes [15-18]. In our simulation of the walk, in order to compile with ZigBee Healthcare profile [3,20] and also satisfying the characteristics of the proposed ZFMN, the end device sends 110 bytes data per second to simulate the data collection-referred as packet inter-arrival time, Ti.

\section{Numerical Results}

In this section, the performance of a ZFMN is evaluated when there are a varying number of end devices $(\mathrm{k})$ moving in the simulated area under the random waypoint mobile model. The investigation includes (Ec) and percentage packet drop (PDR). To quantify the address capacity load of ZFMN, a parameter, namely Initial Address occupancy ratio (Ao), is defined as Ao $=\mathrm{k} / \mathrm{Ec}$.

Figure 4 shows the relationship between $\mathrm{k}, \mathrm{Ec}$ and PDR when Ts $=600 \mathrm{~s}$. Ec indicates the number of addresses left in the entire network (including address left in coordinator and routers) after simulation. Line A (Ec, cal) represents the analytical results given by (3) (e.g. when $\mathrm{k}=4$ and $\mathrm{Na}=80$ (Table 1), $\mathrm{Ec}=80-4=76$ ). The calculated results actually refer to the ideal value of Ec when each end device occupies a single address. Line $\mathrm{B}(\mathrm{Ec}, \mathrm{sim})$ is the simulation results representing the actual amount of idle addresses of the ZFMN. Ec, cal and Ec, sim are compared to determine whether the TAA scheme is efficient in the ZFMN. As shown in Figure 4, in general, Ec drops as $\mathrm{k}$ increases, for both the Ec, cal 


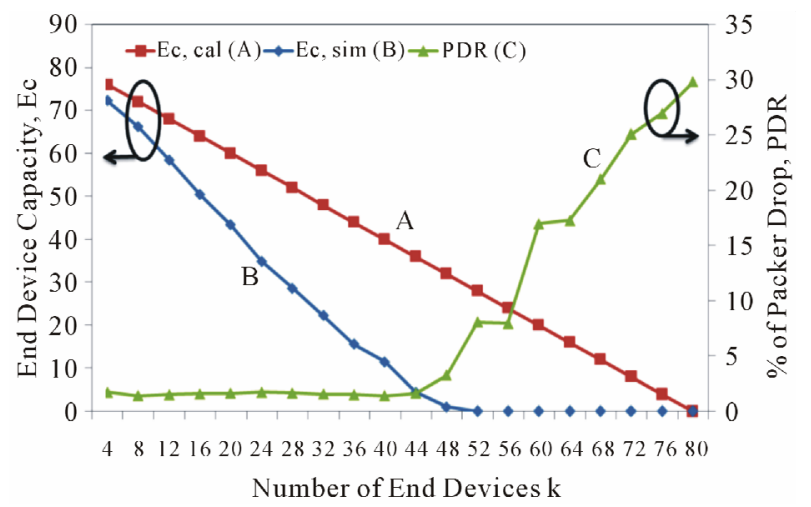

Figure 4. Ec and PDR against $k$ for $T s=600 \mathrm{~s}$.

(A) and Ec, sim (B). Such drop is attributed to the fact that each end device must obtain an address to enable a ZigBee communication. It is observed that Ec, sim dropped more drastic than Ec, cal, indicating that 1) the number of occupied addresses is more than the number of end device; 2) some end devices occupy more than one address. It is also noted that when $\mathrm{k}>48$ (Ao $>60 \%$ ), Ec, sim reaches zero. This implies that no address is available in the ZFMN. The resultant address shortage will thus present a critical threat since address assignment cannot be self-sustained in TAA.

To investigate the deterioration of performance of a ZFMN due to address shortage, PDR against $\mathrm{k}$ is plotted in curve $\mathrm{C}$ in Figure 4. The PDR is low when $\mathrm{k}$ varies from 4 to 44 (Ao $<55 \%$ ) because the ZFMN has not reached address shortage. However, PDR increases dramatically and reaches $30 \%$ when $\mathrm{k}$ gradually increases from 48 to 80 (Ao $>60 \%$ ) because these scenarios have reached the address shortage within $10 \mathrm{~min}$ and thus some end devices will become orphaned. In general, PDR increases when $\mathrm{k}$ increases. Hence Ao $=55 \%$ is the limiting case that needs to draw attention. To gain more insight, Ao $=50 \%(\mathrm{k}=40, \mathrm{Ts}=2400 \mathrm{~s})$ is now investigated (for simplicity reason, Ao $=50 \%$ is used to replace Ao $=55 \%$ ). Typically, medical data are collected several times per day to once a week [3]. Since the ZFMN is mobile in nature, a vigorous test is devised and performed to explore its strength to deal with mobility. Hence the analysis in the present simulation is designed such that the data is collected every second (referred as "1 s collection") from end devices to cater for the most adverse mobility requirement. The " $1 \mathrm{~s}$ collection" is described by the behavior of PDR in a ZFMN (shown in Figure 5). Figure 5 gives an analysis of PDR versus Ec when $\mathrm{k}=40$. When $\mathrm{t}<120 \mathrm{~s}, \mathrm{Ec} \sim=40$ implying that 40 end devices are walking inside the coverage of their first parents (router or coordinator). At $\mathrm{t}=120 \mathrm{~s}, \mathrm{Ec}<40$ because the end devices begin to move out of their first parents' coverage and hand off to new parents as migrants. In such a circumstance, migrated end devices will

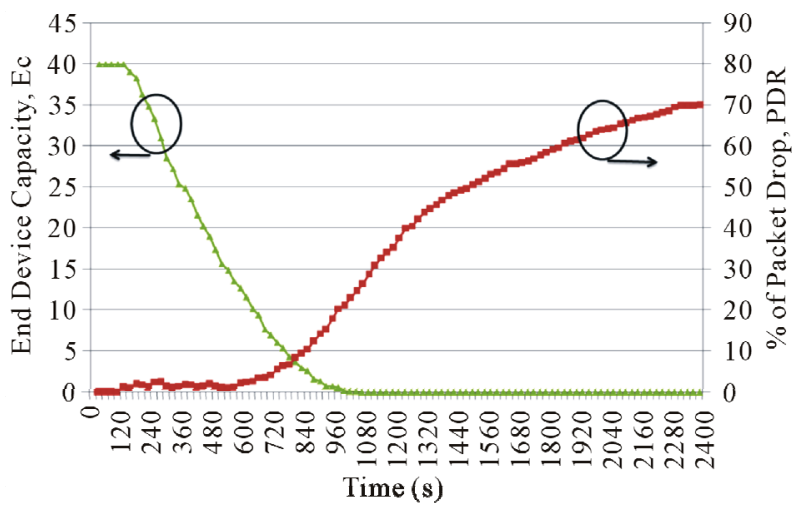

Figure 5. PDR and Ec of scenario 10 when Ts $=2400 \mathrm{~s}$.

request for new addresses. As a result, Ec descends drastically from 40 and vanishes at $\mathrm{t}=960 \mathrm{~s}$. At the same time, PDR increases sharply and reaches $70 \%$ when $\mathrm{t}=$ 2280 s. However, it is important to point out that the PDR $<3 \%$ for $\mathrm{t}<600 \mathrm{~s}$. This implies that the network still functions properly between $\mathrm{t}=240 \mathrm{~s}$ to $\mathrm{t}=600 \mathrm{~s}$. Hence the ZFMN can still manage to provide sufficient address during this "survival" period. From the investigation, it is seen that when PDR $\leq 3 \%$, quality communication has been achieved. The $3 \%$ background PDR is estimated to originate from network congestion and signal degradation which are integral parts of the intrinsic property of the ZFMN. When Ts $=2400 \mathrm{~s}$, it is seen from Figure 5 that PDR has reached its maximum point and Ec is diminishing small. Hence, it is concluded that $\mathrm{t}=$ $600 \mathrm{~s}$ is the limiting case. The intention of this investigation is to point out the potential problem that will be encountered if ZigBee will be employed for mobile health applications. Remedial methods to resolve the address shortage issue is out of the scope in this paper and is desperately required in order to bring ZigBee into real applications.

\section{Problem Statement}

ZigBee is originally designed for non mobile applications. Despite TAA distributes address to each router and coordinates with a predefined amount, the address shortage can be avoided by designing a ZFMN using appropriate network parameters such as $\mathrm{Cm}$, Rm and Lm. However, the number of children node of each routers and coordinator cannot be predicted accurately in a ZFMN because an end device is nomadic in nature and constantly move from coverage to coverage and from time to time. Mobile end devices request new address from routers or the coordinator upon joining new parents, rendering a single mobile end device acquires multiple addresses typically. Thus it is not uncommon that address shortage often occurs and cannot be avoided in a ZFMN network design. 


\section{Conclusion}

The mobile health application will gain popularity in the near future. Since ZigBee offers the mesh feature, it will be a potential candidate as the technology platform for deployment. Existing ZigBee Health Care Profile integrates into IEEE 11073 and is only defined for fixed network. The mobile health application demands mobility which is not resolved under current profile and standard. A ZigBee mobility management profile has been defined to provide mobility in a fixed ZigBee network. The network is referred as ZigBee Fixed Mobile Network (ZFMN) which has characteristics different to a ZigBee mobile ad hoc network. A common area of size of 20,000 square feet has been studied in accordance with the norm of recommendation from ZigBee Alliance. As a result, a pool of devices comprising a coordinator, three routers and eighty end devices are required for such an area. Analysis of network performance using OPNET reveals that there is a problematic address shortage issue which prohibits mobility. It is evaluated that the network may survive address shortage (communicate efficiently) when the packet drop rate (PDR) is maintained below $3 \%$. In order to cater for the most adverse mobility requirement, a vigorous test has been devised and performed by simulating data collection in $1 \mathrm{~s}$ (referred as " $1 \mathrm{~s}$ collection"). It is found that the background PDR $<3 \%$ and the ZFMN performs satisfactorily. Thus it is asserted in this report that PDR $<3 \%$ is the condition to provide reasonable QoS for mobile health applications such as patient monitoring system using ZFMN. Remedial solution to avoid address shortage is out of the scope in this paper and this is left as an open question for further research.

\section{REFERENCES}

[1] W.-H. Chung, P.-C. Hsiu, Y.-Y. Shih, A.-C. Pang, Y.-K. Huang and K.-C. Hung, "Mobility-Robust Tree Construction in ZigBee Wireless Networks," The Proceeding of 2011 IEEE International Conference on Communication, 5-9 June 2011, pp. 1-6.

[2] H. Dhaka, A. Jain and K. Verma, "Impact of Coordinator Mobility on the Throughput in a ZigBee Mesh Networks," The Proceeding of 2010 IEEE 2nd International Advance Computing Conference (IACC), 19-20 February 2010, pp. 279-284.

[3] J. S. Mu and K. H. Liu, "A Study on the Routing Selection Method in Zigbee Networks Based on the Mobility of the Nodes and the Scale of the Network," The Proceeding of 2010 International Conference on Communications and Mobile Computing (CMC), Vol. 3, 2010, pp. 405-409.

[4] T. Hirou, Convergence Wireless Lars Schmitt, Philips, "ZigBee Health Care-What's in Store for Health Care using ZigBee?” ZigBee Alliance, 2009.

https://docs.zigbee.org/zigbee-docs/documents
[5] J. Adams, "ZigBee Health Care Wireless Control and Sensing in Health Care, Sports and Fitness, and Wellness," ZigBee Alliance, 2009. https://docs.zigbee.org/zigbee-docs/documents

[6] ZigBee Alliance, "ZigBee Health Care Profile Specification," Revision 15, Version 1.0, March 2010.

[7] D. I. Shin, S. J. Huh and P. J. Pak, "Patient Monitoring System Using Sensor Network Based on the ZigBee Radio," 6th International Special Topic Conference on ITAB, Seoul, 8-11 November 2007, pp. 313-315.

[8] P. Frehill, D. Chambers and C. Rotariu, "Using Zigbee to Integrate Medical Devices," Proceedings of the 29th Annual International Conference of the IEEE EMBS, 22-26 August 2007, pp. 6717-6720.

[9] W.-W. Lin and Y.-H. Sheng, "Using OSGi UPnP and Zigbee to Provide a Wireless Ubiquitous Home Healthcare Environment," The proceedings of the Second International Conference on Mobile Ubiquitous Computing, Systems, Services and Technologies, 2008, Valencia, 29 September-4 October 2008, pp. 268-273.

[10] S. Nourizadeh, C. Derussent, Y. Q. Song and J. P. Thomesse, "Medical and Home Automation Sensor Networks for Senior Citizens Telehomecare," The Proceeding of the First International Workshop on Medical Applications, 2009.

[11] J. Hou, B. Chang, D.-K. Cho, and M. Gerla, "Minimizing 802.11 Interference on Zigbee Medical Sensors," In BodyNets, 2009.

[12] H. S. Kim and J. Yoon, "Hybrid Distributed Stochastic Addressing Scheme for ZigBee/IEEE 802.15.4 Wireless Sensor Networks," ETRI Journal, Vol. 33, No. 5, 2011, pp. 704-711. doi:10.4218/etrij.11.0110.0501

[13] L.-H. Yen and W.-T. Tsai, "The Room Shortage Problem of Tree-Based ZigBee/IEEE 802.15.4 Wireless Networks," Computer Communications, Vol. 33, No. 4, 2010, pp. 454-462. doi:10.1016/j.comcom.2009.10.013

[14] OPNET University Program. http://www.opnet.com/services/university/

[15] N.-C. Liang, P.-C. Chen, T. Sun, G. Yang, L.-J. Chen, and M. Gerla, "Impact of Node Heterogeneity in ZigBee Mesh Network Routing," IEEE International Conference on Systems, Man and Cybernetics, 8-11 October 2006, pp. 187-191. doi:10.1109/ICSMC.2006.384380

[16] T. J. Kwon, M. Gerla, V. K. Varma, M. Barton and T. R. Hsing; "Efficient Flooding with Passive Clustering-An Overhead-Free Selective forward Mechanism for Ad Hoc/Sensor Networks," The IEEE Proceedings, Vol. 91 No. 8, 2003, pp. 1210-1220. doi:10.1109/JPROC.2003.814920

[17] B. Sun, K. Wu, Y. Xiao and R. H. Wang, "Integration of Mobility and Intrusion Detection for Wireless ad Hoc Networks," International Journal of Communication Systems, Vol. 20, No. 6, 2007, pp. 695-721. doi: $10.1002 /$ dac. 853

[18] A. S. Arezoomand and M. Pourmina, "Prolonging Network Operation Lifetime with new Maximum Battery Capacity Routing in Wireless Mesh Network," 2010 The 2nd International Conference on Computer and Automa- 
tion Engineering (ICCAE), Singapore, 26-28 February 2010, pp. 319-323.

[19] ZigBee Alliance, "ZigBee Stack Profile: Platform Restrictions for Compliant Platform Testing and Interoperability," Revision 9, January 2008.
[20] "ISO/IEC/IEEE Health Informatics-Personal Health Device Communication-Part 20601: Application Profile -Optimized Exchange Protocol," ISO/IEEE 1107320601:2010, 2010, pp. 1-208. 